\title{
Reaction-in-Flight Neutrons as a Test of Stopping Power in Degenerate Plasmas
}

\author{
A.C. Hayes, Gerard Jungman, A.E. Schulz, M. Boswell, M.M. Fowler, \\ G. Grim, A. Klein, R.S. Rundberg, J.B. Wilhelmy, D. Wilson \\ Los Alamos National Laboratory, Los Alamos, NM, USA 87545 \\ C. Cerjan, D. Schneider, A. Tonchev, C. Yeamans \\ Lawrence Livermore National Laboratory, Livermore, CA, 94551
}

\begin{abstract}
We present the first measurements of reaction-in-flight (RIF) neutrons in an inertial confinement fusion system. The experiments were carried out at the National Ignition Facility, using both Low Foot and High Foot drives and cryogenic plastic capsules. In both cases, the high-energy RIF $\left(E_{n}>15 \mathrm{MeV}\right)$ component of the neutron spectrum was found to be about $10^{-4}$ of the total. The majority of the RIF neutrons were produced in the dense cold fuel surrounding the burning hotspot of the capsule and the data are consistent with a compressed cold fuel that is moderately to strongly coupled $(\Gamma \sim 0.6)$ and electron degenerate $\left(\theta_{\text {Fermi }} / \theta_{e} \sim 4\right)$. The production of RIF neutrons is controlled by the stopping power in the plasma. Thus, the current RIF measurements provide a unique test of stopping power models in an experimentally unexplored plasma regime. We find that the measured RIF data strongly constrain stopping models in warm dense plasma conditions and some models are ruled out by our analysis of these experiments.
\end{abstract}

\section{INTRODUCTION}

At the National Ignition Facility (NIF)[1], high yield inertial confinement fusion (ICF) plasmas are studied using cryogenically cooled capsules containing an equimolar mixture of deuterium and tritium (DT). The cryogenic capsule designs involve three distinct material layers: an outer hydrocarbon ablator layer, a thick layer of solid DT ice, and a central sphere of DT gas. The capsule is compressed to sufficient temperatures and densities that burn is initiated in the central gas region with a design that promotes the propagation of a thermal wave into the surrounding dense cold DT fuel. Though burn propagation into the cold fuel has yet to be achieved, the assembled configuration of the compressed capsule is suitable for the production of rare nuclear reactions with measurable yields. One such process, the production of reactionin-flight or tertiary neutrons, is the central focus of the present work. We examined implications of the measured yield of these high-energy neutrons within the context of different theoretical stopping power models.

Reaction-in-Flight (RIF) neutrons require three successive reactions. First, a primary $14.1 \mathrm{MeV}$ DT neutron is produced; second, this high energy neutron then undergoes elastic scattering with deuterium or tritium ions in the plasma, energetically up-scattering these ions to a range of energies from 0 to more than $10 \mathrm{MeV}$. In the third step, the energetic knock-on ion undergoes a DT reaction with a thermal ion in the plasma, producing a continuous spectrum of RIF neutrons in the energy range 9.2-30 MeV. The process of RIF production is summarized in Figure 1. Knock-on ions lose energy as they traverse the plasma, which directly affects the number and energy spectrum of the produced RIF neutrons. Thus, this sensitivity can be used to extract information about plasma stopping powers. As detailed below, in the case

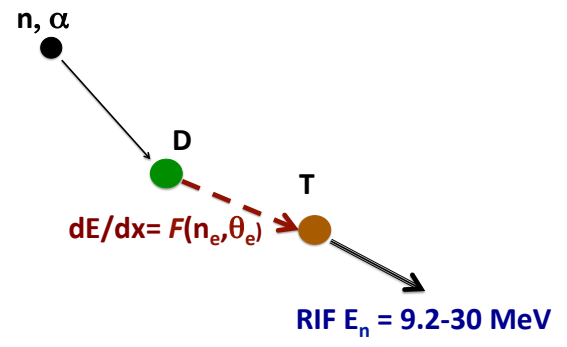

FIG. 1. RIF production involves three consecutive steps. First, a primary neutron and alpha particle are produced in a DT fusion reaction. Either of these can then knock a $\mathrm{D}$ or $\mathrm{T}$ ion up to $\mathrm{MeV}$ energies through elastic scattering. The knock-on ion then undergoes a secondary DT fusion reaction producing a RIF neutron. The specific RIF diagnostic neutrons in the present work are those measured by the

${ }^{169} \operatorname{Tm}(n, 3 n)$ reaction, for which $E_{n}>15 \mathrm{MeV}$. This Tm diagnostic is dominated by the high-energy neutron-induced RIF since the alpha-induced RIFs only contribute about $10 \%$ of the total thulium signal.

of cryogenic NIF capsules, RIFs probe stopping under previously unexplored plasma conditions.

\section{RIF PRODUCTION IN CRYOGENIC NIF CAPSULES}

The RIF production depends directly on the fluence of knock-on ions in the plasma. Under plasma conditions in which they can escape the capsule, knock-on ions have been successfully measured and characterized, including 
their partial down-scattering [2]. The magnitude of the initial knock-on fluence $Q_{0}$ is determined by the plasma burn conditions, while the spectral shape, $q_{0}\left(E_{0}\right)$, is determined simply by the kinematics of the elastic collisions between the $14 \mathrm{MeV}$ neutrons and the plasma ions. The magnitude of the knock-on fluence is controlled by the 14 $\mathrm{MeV}$ neutron fluence, $\phi_{n}$, the number density of DT ions in the plasma, $\hat{n}_{D T}$, and the knock-on cross section $\sigma_{K O}$, i.e., $Q_{0}=\phi_{n} \hat{n}_{D T} \sigma_{K O}$. Thus, a capsule with a high 14 $\mathrm{MeV}$ fluence and a high DT density is needed to ensure a high knock-on fluence. For this purpose, the cryogenic designs are ideal: they provide a strong neutron source (currently up to $10^{16}$ total neutron number) from the burning hotspot, and a high number density $\left(10^{25}-10^{26}\right.$ $\mathrm{cm}^{-3}$ ) of DT ions in the cold fuel. From these simple considerations, we expect the RIF production to take place predominantly in the cold fuel, and this expectation is realized in the detailed simulations discussed below.

Transport of the knock-on ions in the plasma results in a change from the initial shape $q_{0}\left(E_{0}\right)$ and magnitude $Q_{0}$ of the spectrum, a change that is determined by the form of the stopping power. If the stopping length of the knock-on ions is short compared to the spatial variations in the temperature and density within the plasma, the differential knock-on fluence can be related [3] to the energy loss, $d E / d x$, and the initial knock-on fluence by

$$
\frac{d \psi_{k o}}{d E_{k o}}\left(E_{f}\right)=\frac{Q_{0}}{\left|d E / d x\left(E_{f}\right)\right|} \int_{E_{f}}^{E_{0 \max }} d E_{0} q_{0}\left(E_{0}\right) .
$$

From equation 1, it follows that determination of the knock-on fluence in the plasma is inversely related to the stopping power. In Figure 2 we show the shape of the knock-on fluence for deuterons in a plasma with constant density and temperature, $n_{e}=10^{26} \mathrm{~cm}^{-3}$ and $\theta_{e}=0.2$ $\mathrm{keV}$, using three different stopping power models and eq. 1. The upper curve uses the Maynard-Deutsch [4] stopping power as parameterized by Zimmerman [5], while the dashed curve uses the same model but neglects electron degeneracy. Electron degeneracy lowers the magnitude of the stopping, increasing the knock-on fluence. We discuss the effects of degeneracy in more detail in the next section. Finally, the lowest curve in Figure 2 shows the effect of using a purely classical stopping model [6]; as can be seen, quantum effects (including degeneracy) increase the knock-on fluence by more than a factor of two over classical predictions.

We note that, in general, it is not necessary to invoke the assumption that the stopping lengths are short, and our full simulations make no such assumption. Nonetheless, eq. 1 is very useful in illuminating the relationship between the stopping power and knock-on fluence. The knock-on fluence, and hence the stopping, can be determined by measuring the yield of nuclear reactions involving knock-on particles [7. In the present work we accomplish this goal by directly measuring the RIF neutrons.

The RIF production per volume is determined by the integral of the knock-on fluence over the DT cross section,

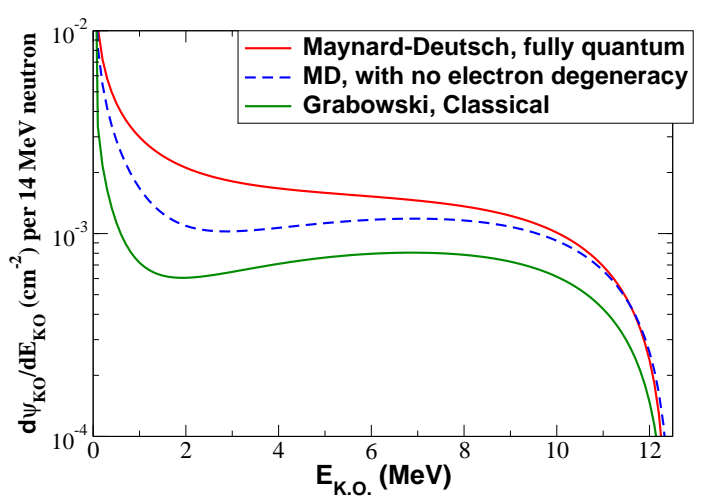

FIG. 2. The knock-on fluence for deuterons in a plasma with constant density and temperature, $n_{e}=10^{26} \mathrm{~cm}^{-3}$ and $\theta_{e}=0.2 \mathrm{i} \mathrm{keV}$, using different stopping power models and eq. 1 The uppermost curve uses a fully quantum stopping model [4], while the dashed curve uses the same model but neglects electron degeneracy. The lowest curve is the prediction from a purely classical stopping model [6]. Quantum effects (including degeneracy) increase the knock-on fluence by more than a factor of two over classical predictions.

weighted by the density of DT ions $[\underline{8}, 9$,

$$
\frac{d \Gamma_{R I F}}{d E_{R I F} d V}=n_{D T} \int_{0}^{E_{k o}^{\max }} \frac{d \psi_{k o}}{d E_{k o}} \sigma_{D T} \frac{d F}{d E_{R I F}} d E_{k o}
$$

The factor $d F / d E_{R I F}$ is the kinematic factor needed to ensure that a given knock-on energy produces a RIF neutron within the measured energy window, which for the present experiments is the window $E_{R I F}>15 \mathrm{MeV}$. Again, because the density of DT ions is highest in the cold fuel, the majority of RIFs are produced in this region of a cryogenic capsule.

The RIF production at peak burn, predicted from 1-D [10] and 3-D [1] simulations, originates in the shocked cold fuel with fewer than $10 \%$ produced in the hot spot. Figure 3 presents plots of the simulated spatial dependence of the energy deposition of the knock-on deuterons from a 3-D HYDRA [1] simulation. The majority of deuterons deposit their energy in the cold fuel. For comparison, we show the position of alpha-particle energy deposition, which is entirely contained in the hotspot of the capsule. While both experiment and simulations show capsule-to-capsule variations in the neutron yield and the density and temperature of the cold fuel, the situation depicted in Figure 3 is representative of the conditions in cryogenic capsules.

\section{THE PLASMA COUPLING AND STOPPING MODELS}

At peak capsule compression, the outer dense DT fuel is at a temperature of a fraction of a $\mathrm{keV}$ and a den- 

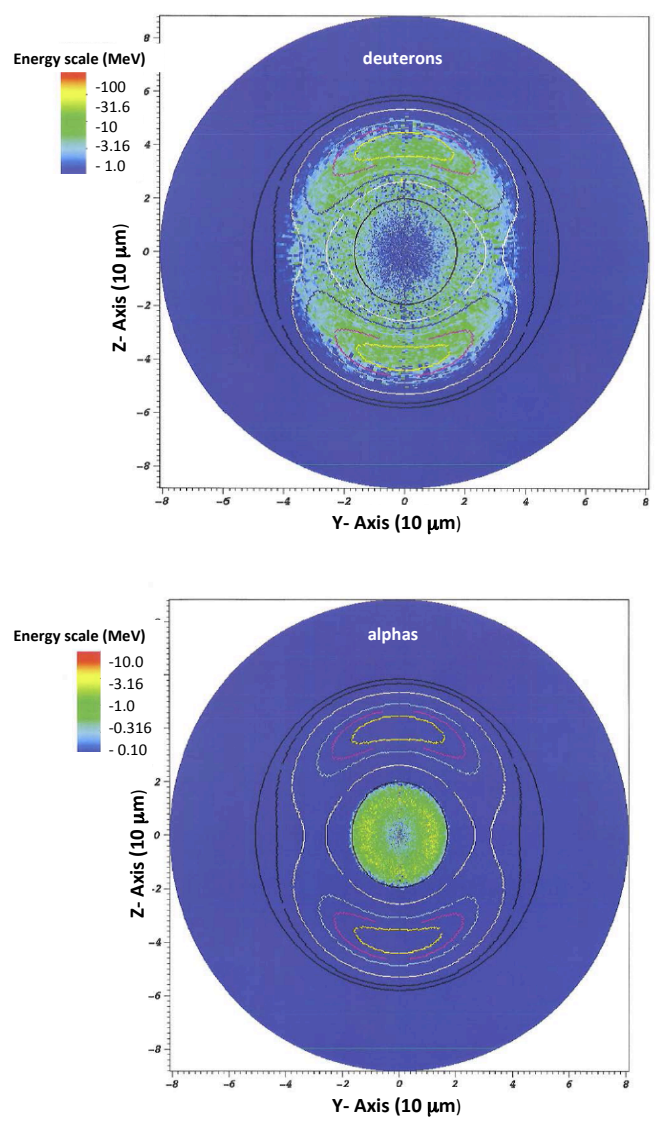

FIG. 3. The predicted spatial dependence of the energy deposition of the knock-on deuterons and alphas from a 3-D HYDRA [1] simulation with superimposed density contours for a typical cryogenic capsule implosion relevant to the present RIF measurements. The knock-on deuterons deposit their energy predominantly in the cold fuel. In sharp contrast, the alpha particles deposit all of their energy at the edge of the hotspot.

sity that is approaching $10^{26} \mathrm{~cm}^{-3}$. At these densities and temperatures, some standard stopping models, such as the Brown, Preston and Singleton (BPS) model [12, become inaccurate because such models are designed for and restricted to weakly-coupled plasmas. The plasma coupling parameter is defined as the ratio of the potential energy to the plasma temperature, $\Gamma=Z e^{2} / \theta R$, where $Z$ is the charge of the moving ion, $\theta$ is the plasma temperature, and $R$ is a radius that roughly describes the distances between charges in the plasma. Different choices for $R$ are found in the published literature, with the Wigner or Debye radius being the most common choices.

Loosely speaking, low densities and high temperatures correspond to weakly coupled plasmas in which longdistance collective effects dominate, while high density, low temperature plasmas tend to be strongly coupled. For small values of $\Gamma(\Gamma<0.1)$ and non-degenerate plasmas, there are several stopping models that are known to be accurate, see for example [4, 12, 13. Though the the- oretical framework used is quite different in each of these treatments, the derived stopping powers can be shown [14 to be numerically identical under weakly-coupled conditions. As the coupling $\Gamma \rightarrow 1$, though, the plasma becomes strongly coupled. An additional important characteristic of the plasma that affects the form of the energy loss is the degree of electron degeneracy which is determined by the ratio of the electron temperature $\theta_{e}$ to the Fermi energy $\theta_{F}$. For a degenerate plasma, the temperature determining the stopping power is an effective temperature, $\theta_{\text {eff }}=\frac{3}{5} \theta_{F} F\left(\theta_{e}\right)$. The function $F\left(\theta_{e}\right)$ is obtained by solving for the chemical potential of the system and has the property that in the zero temperature limit, $\theta_{\text {eff }} \rightarrow \frac{3}{5} \theta_{F}$, while in the high temperature limit $\theta_{\text {eff }} \rightarrow \theta_{e}$. Under moderately to strongly coupled conditions, $\Gamma>0.3$, published models of plasma stopping powers disagree, especially since not all the models include degeneracy effects. To date, there have been no experimental tests of plasma stopping powers for non-weakly coupled and degenerate plasmas. However, the cold fuel of cryogenic NIF capsules do provide a system with such conditions, hence the RIF measurements probe stopping powers in a previously unexplored plasma regime.

Figure 4 contains a plot of the ratio of the Fermi temperature to the actual temperature at peak burn time as function of the capsule radius, as predicted in a 1-D HYDRA simulation for a standard High Foot 15 cryogenic capsule implosion. As can be seen, the cold fuel is degenerate, with the electron temperature being a factor of 3-5 below the Fermi temperature. The cold fuel is divided into an inner shocked and outer unshocked region, the inner shocked region being higher in density and temperature.

Figure 5 displays the effective temperature and the ratio $\rho /\left(\theta_{\text {eff }}^{3 / 2}\right)$. This ratio controls the magnitude of the stopping power, which in the case of stopping by electrons, can be parameterized as

$$
\frac{d E}{d X}=-\frac{4 \pi Z_{p}^{2} e^{4}}{m_{e} v_{p}^{2}}\left(\frac{n_{e}}{\theta_{e}}\right) G(y) \ln \left(\Lambda_{e}\right),
$$

where $v_{p}$ and $Z_{p}$ are the velocity and charge of the projectile, $n_{e}(\equiv \rho)$ is the electron density, and $G\left(y=\frac{m_{e} E_{p}}{m \theta_{e}}\right)$ is a function controlling the shape of the stopping [4, 16]. For small $y, G(y) \sim y^{1 / 2}$ and the magnitude of the stopping power is set by the ratio $n_{e} /\left(\theta_{\mathrm{eff}}^{3 / 2}\right)$. In a fully degenerate system, the effective temperature is fully determined by the density, and the ratio $n_{e} /\left(\theta_{\text {eff }}^{3 / 2}\right)$ is a fixed physical constant. For this reason, the stopping power is more constrained in the cold fuel, and a cold fuel temperature measurement is not necessary. This is in strong contrast to a weakly coupled system.

\section{THE EXPERIMENTS}

The signal from RIFs is generally expected 17 to be small $\left(\sim 10^{-4}\right)$ relative to the primary $14 \mathrm{MeV}$ sig- 


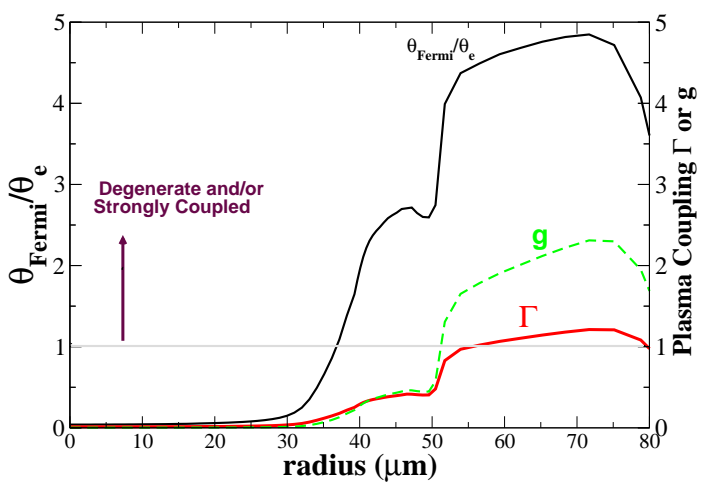

FIG. 4. The ratio of the Fermi temperature to the electron temperature (black curve) as a function of the capsule radius at peak burn time from a 1-D HYDRA simulation. In the cold fuel $(\mathrm{CF})(r \sim 40-80 \mu \mathrm{m})$, the plasma is electron degenerate. Also shown are two standard definitions of the plasma coupling, $\Gamma=\frac{Z e^{2}}{\theta R_{W}}$ (red curve) and $g=\frac{Z e^{2}}{\theta R_{D}}$ (green curve), where $R_{W}$ and $R_{D}$ are the Wigner and Debye radii respectively. The cold fuel ranges from moderately coupled to strongly coupled. At peak burn the simulations predict that the $\mathrm{CF}$ has two distinct regions with different degrees of degeneracy and coupling, the region $r \sim 40-50 \mu \mathrm{m}$ within the shocked $\mathrm{CF}$ and the region $r \sim 50-80 \mu \mathrm{m}$ upstream of the shock. The capsule hydrocarbon ablator material starts at and extends beyond a radius of $80 \mu \mathrm{m}$.

nal. Thus, experimental detection requires measuring the neutron spectrum above the thermally broadened primary $14 \mathrm{MeV}$ peak. In the experiments reported here, RIF detection was achieved at NIF by measuring neutrons with energies above $15 \mathrm{MeV}$ via neutron activation of thulium foils. The $(n, 3 n)$ reaction on natural thulium $\left({ }^{169} \mathrm{Tm}\right)$ is a threshold reaction, requiring $E_{n}>15 \mathrm{MeV}$ and producing ${ }^{167} \mathrm{Tm}$. The experiments were designed to search for the presence of ${ }^{167} \mathrm{Tm}$, which decays with a half-life $\tau_{1 / 2}=9.25$ days by electron capture to ${ }^{167} \mathrm{Er}$, with the emission of a $207.79 \mathrm{keV} \gamma$-ray $41 \%$ of the time. Thulium foils were placed in the Solid Radiochemistry Collector (SRC) holders that are attached to a Diagnostic Instrument Manipulator (DIM) in the NIF target chamber [18. The SRC holders are $50 \mathrm{~cm}$ from the ICF target, and two Tm foils were used, one in a holder on a DIM in the equatorial direction and one in the polar direction. Thulium foils of thickness $2.0 \mathrm{~mm}$ or $0.5 \mathrm{~mm}$ were used in the experiments depending on the shot. The quantitative measurement of ${ }^{168} \mathrm{Tm}$ provided a consistency check of the primary $14 \mathrm{MeV}$ neutron production that was determined independently by several direct time-of-flight measurements, as well as other activation techniques.

The primary experimental challenge for the ${ }^{167} \mathrm{Tm}$ measurements was the huge background of $\gamma$-rays pro-

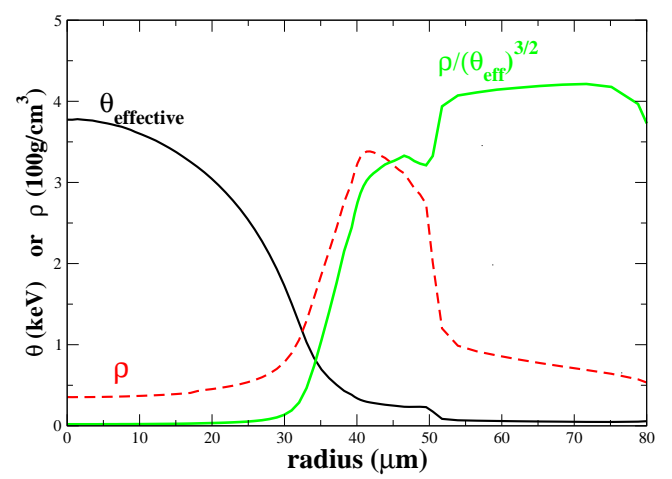

FIG. 5. The effective temperature and the density at peak burn time, as a function of the DT fuel radius, from a 1-D HYDRA simulation. The ratio $n_{e} /\left(\theta_{\text {eff }}^{3 / 2}\right)$ is approximately constant in each region of the CF. It is this combination of density and effective temperature that determines the stopping power at low knock-on energies. The electron degeneracy and the corresponding approximately constant value of $n_{e} /\left(\theta_{\text {eff }}^{3 / 2}\right)$ in the CF greatly reduce the uncertainty with which we can extract $d E / d X$ in the absence of a CF temperature measurement.

duced in the activation of the foils by the much higher fluence of primary $14 \mathrm{MeV}$ neutrons, in particular those from ${ }^{168} \mathrm{Tm}\left(\tau_{1 / 2}=93.1\right.$ days $)$. Thus, suppression of background gamma-rays was crucial in the experiments. To achieve this suppression, the activated foils were shipped to Los Alamos (LANL), where two clover detectors were deployed to assay the ${ }^{168} \mathrm{Tm}$ and ${ }^{167} \mathrm{Tm}$ activity in the foils. The LANL clover system consists of two high efficiency clover Ge detectors. Each clover consists of 4 segmented high purity Ge crystals, surrounded by an active NaI Compton suppressor. The ${ }^{167} \mathrm{Tm}$ decays to a $208 \mathrm{keV}$ isomeric level in ${ }^{167} \mathrm{Er}$ that has a half-life of 2.28 sec. This level decays $100 \%$ of the time to the ${ }^{167} \mathrm{Er}$ ground state. Since there is nothing in coincidence with this transition, this decay sequence is ideal for identification by our segmented clover detector. In contrast, the major background from the decay of ${ }^{168} \mathrm{Tm}$ has a highly complex decay sequence that has a high probability of interacting with multi-elements in the clover array. These coincident events can be readily removed from the event stream, resulting in a greatly improved ${ }^{167} \mathrm{Tm}$ signal-tonoise ratio. Details of the setup are presented in [19].

The first positive RIF signals were seen in March of 2013 for the NIF shot N130331. These signals were obtained from a cryogenic implosion in which the protocol was a design from the National Ignition campaign (NIC), sometimes referred to as a Low Foot design [1]. The measured average ${ }^{167} \mathrm{Tm} /{ }^{168} \mathrm{Tm}$ ratio for the two 2 $\mathrm{mm}$ foils was $(2.0 \pm 0.74) \times 10^{-5}$, which corresponds to a 


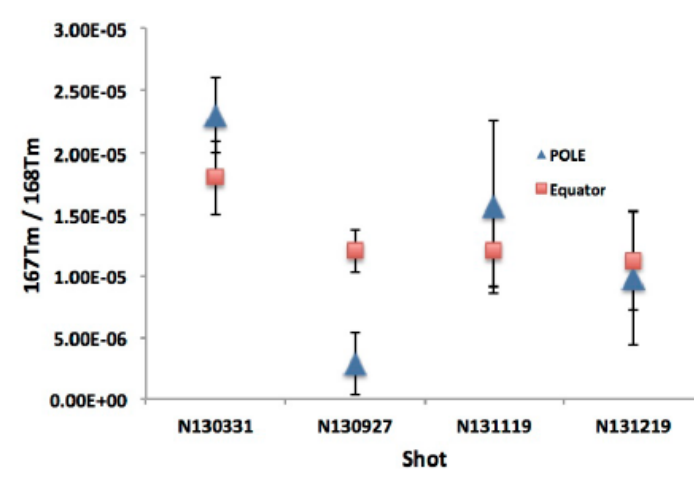

FIG. 6. The measured ${ }^{167} \mathrm{Tm} /{ }^{168} \mathrm{Tm}$ ratios for the four shots which used the thicker $(2 \mathrm{~mm})$ foils. The corresponding $\operatorname{RIF}\left(E_{n}>15 \mathrm{MeV}\right) /$ Total ratios are slightly shot dependent but are about 8 times larger than the Tm ratios. The factor of 8 comes from the difference in the shape of the $14 \mathrm{MeV}$ and RIF spectra and the $(n, 2 n)$ and $(n, 3 n)$ cross sections for Tm. The shot dependence arises since the shape of the RIF spectrum depends on the density of the cold fuel.

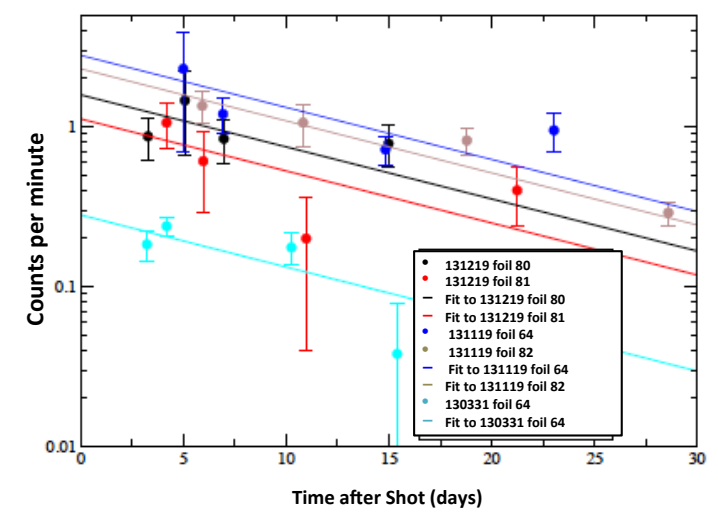

FIG. 7. The $208 \mathrm{keV}$ signal for several shots as a function of the time since the shot. The decay rates of the signals are consistent with the 9.25 day half-life of ${ }^{167} \mathrm{Tm}$.

$\operatorname{RIF}\left(E_{n}>15 \mathrm{MeV}\right) /$ total neutron ratio of $10^{-4}$. The measured ${ }^{167} \mathrm{Tm} /{ }^{168} \mathrm{Tm}$ atom ratio is the integral of the $\mathrm{RIF} /$ total spectrum against the respective Tm isotopic production cross sections. Since these cross sections are adequately known, the integral primary RIF spectrum can be deduced. During 2013, three additional RIF measurements were performed using $2 \mathrm{~mm}$ Tm foils, and the results were in the $1-2 \times 10^{-5}$ range, with an uncertainty of about $35 \%$. These three additional shots were High Foot design [15] capsules. The results for the four $2 \mathrm{~mm}$ foils are summarized in Figure 6. The measured decays of the $208 \mathrm{keV}$ signal in all of these experiments were consistent with the known ${ }^{167} \mathrm{Tm}$ half life (Fig. 7).

It is worth noting that for shot N130927 we saw a

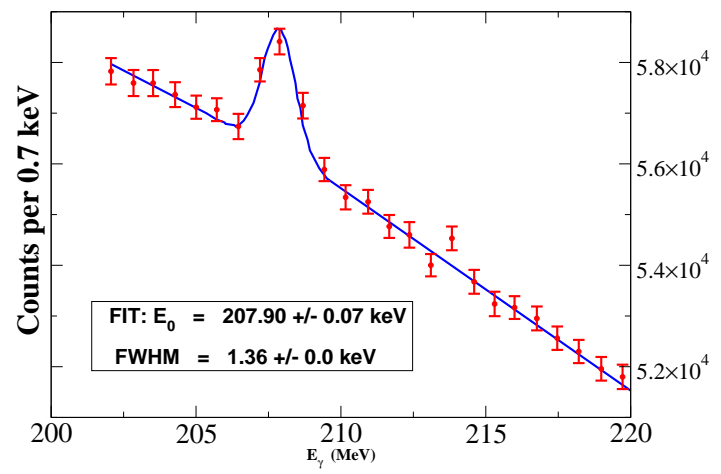

FIG. 8. The $208 \mathrm{keV}$ peak from the decay of ${ }^{167} \mathrm{Tm}$ for the shot N140304. The corresponding measured ratio of ${ }^{167} \mathrm{Tm} /{ }^{168} \mathrm{Tm}$ was $1.69 \pm 0.24 \times 10^{-5}$.

large asymmetry between the signal measured in the polar versus the equatorial directions, where the foil in the equatorial direction showed a ${ }^{167} \mathrm{Tm} /{ }^{168} \mathrm{Tm}$ ratio of $1.25 \pm 0.3 \times 10^{-5}$, while the polar signal was consistent with zero, $2.5 \pm 2.5 \times 10^{-6}$. The neutron image of the cold fuel for this same shot was also highly asymmetric and it is not clear that the cold fuel was well assembled. This unexpectedly large asymmetry is still under theoretical investigation and we did not include this shot in our analysis of the stopping powers.

The approximate $35 \%$ uncertainty in the RIF signal seen in the $2 \mathrm{~mm}$ thick foils was largely due to selfattenuation of the $208 \mathrm{keV} \gamma$-ray signal within the foils, which limited the signal statistics. For this reason we decided to move to thinner, $0.5 \mathrm{~mm}$, foils. Typically, the thinner foils reduced the uncertainties to about $15 \%$. Figure 8 shows the measured $208 \mathrm{keV}$ peak for one such shot, shot N140304. Here the measured ratio of ${ }^{167} \mathrm{Tm} /{ }^{168} \mathrm{Tm}$ was $1.69 \pm 0.24 \times 10^{-5}$. This lower uncertainty greatly reduces the uncertainty in extracting information on the stopping power in the cold fuel, so we will concentrate on this shot in our analysis below. Again, the decay of the $208 \mathrm{keV}$ signals seen in the $0.5 \mathrm{~mm}$ foils is consistent with the half-life of ${ }^{167} \mathrm{Tm}$, Figure 9.

\section{EXTRACTING LIMITS ON THE STOPPING POWER FROM THE HIGHER PRECISION THIN FOIL RIF MEASUREMENTS}

We first restrict our discussions to the cold fuel. We will add the contributions to the RIF production from the hotspot in the full analysis below. Since the primary $14 \mathrm{MeV}$ neutrons are only produced in the hot spot, the fluence of these neutrons at any position in the cold fuel approximately follows a $1 /\left(4 \pi R^{2}\right)$ law. Thus, the volume and time integral of eq. 2 within the cold fuel can be 


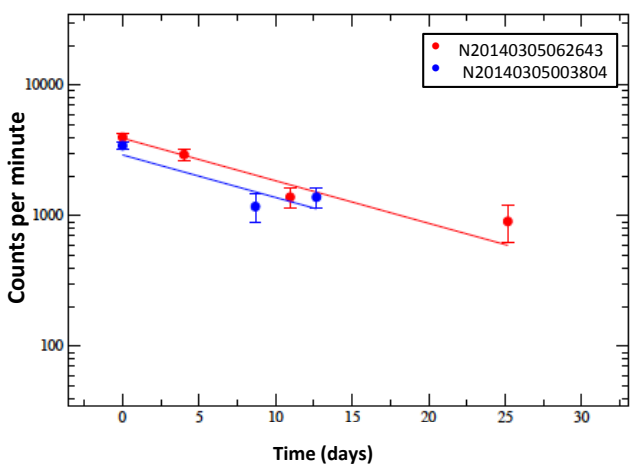

FIG. 9. The decay of the $208 \mathrm{KeV}$ signals from the foils for shots N140305, both consistent with the half-life of ${ }^{167} \mathrm{Tm}$.

written as

$$
N_{R I F}^{C F}=N_{14}\langle\rho R\rangle_{C F} \int_{0}^{E_{k o}^{\max }} \frac{d \tilde{\psi}_{k o}}{d E_{k o}} \sigma_{D T} \frac{d F}{d E_{R I F}} d E_{k o}
$$

where $\frac{d \tilde{\psi}_{k o}}{d E_{k o}}$ is the knock-on fluence per unit primary 14 $\mathrm{MeV}$ neutron, $N_{14}$ is the total $14 \mathrm{MeV}$ yield, and $\langle\rho R\rangle_{C F}$ is the areal density of the cold fuel. In constraining stopping powers from the RIF measurements, our philosophy has been to use as much additional experimental information as possible. Thus, eq. 4 has the advantage of displaying the explicit dependence on the yield and the areal density of the cold fuel. The additional shot-dependent physics determining the RIF ratio is contained in variations of the density (and the related effective temperature) across the cold fuel, as well as possible 3-D cold fuel asymmetries.

In our analyses we used full 3-D simulations constrained 20] by a broad range of experimental data. The constraints involve a physically consistent description of the imploded capsule at stagnation. This method attempts to validate the model of the capsule through stringent comparisons between the radiation hydrodynamic code simulations and a suite of x-ray and neutron imaging data. We carried out a second independent analysis of the RIFs using the 1-D code CPT-Implode, which also focuses on describing the stagnation properties of the capsule correctly. In both cases, we varied the stopping models and examined the change in the shape of the predicted RIF spectrum and its effect on the ${ }^{167} \mathrm{Tm} /{ }^{168} \mathrm{Tm}$ ratio. We also calculated the RIF spectrum using eqs. 1 and 2 , using the density and temperature profiles from the 1D simulation. The three methods agreed to about $20 \%$, providing confidence in the formalism and the chargeparticle transport packages in the codes.

In Table 1, we list the measured and predicted values for the ${ }^{167} \mathrm{Tm} /{ }^{168} \mathrm{Tm}$ diagnostic for two shots, comparing different stopping model predictions. As can be seen, the measurements do not agree with either the CormanSpitzer [21, 22] or Grabowski [6] models. The higher
TABLE I. Comparison between the measured and predicted RIF signals for two shots.

\begin{tabular}{c|c}
\hline $\begin{array}{c}1^{s t} \text { RIF observation } \\
\text { Shot N130331 }\end{array}$ & $167 / 168 \mathrm{Tm}$ \\
\hline Measurement & $2.03 \pm 0.75 \times 10^{-5}$ \\
(averaged over both foils) & $1.99 \times 10^{-5}$ \\
Maynard-Deutsch [4] & $1.57 \times 10^{-5}$ \\
Li-Petrasso [16] & $0.55 \times 10^{-5}$ \\
Corman-Spitzer [21, 22] & \\
\hline Most accurate measurement to-date & $167 / 168 \mathrm{Tm}$ \\
\hline Shot N140304 & $1.69 \pm 0.24 \times 10^{-5}$ \\
\hline Measurement & $1.59 \times 10^{-5}$ \\
(only one foil was used in this shot) & $1.16 \times 10^{-5}$ \\
Maynard-Deutsch [4] & $0.89 \times 10^{-5}$ \\
Li-Petrasso [16] & Model breaks-down \\
Grabowski [6] & $(\Gamma$ too large) \\
BPS [12] & \\
\hline
\end{tabular}

accuracy measurements, which were obtained using the thinner $(0.5 \mathrm{~mm})$ foils, favor the Maynard-Deutsch model over the Li-Petrasso model. The BPS model, which is only designed for weakly coupled plasma, predicts a stopping power with an unphysical sign.

We found that the effect of electron degeneracy on the predicted RIF signal depended on the stopping model. Degeneracy is most important at low knock-on energies, where the stopping power scales with $n_{e} / \theta_{\text {eff }}^{3 / 2}$. The inclusion of electron degeneracy increased the Tm diagnostic anywhere from a factor of 1.5-3.0, depending on the stopping model. A more precise measure of the effect of degeneracy would be afforded by a direct measurement of the shape of the RIF spectrum by neutron time-of-flight. We expect the cold fuel degeneracy to cause a distortion of the spectrum, with an enhancement at lower energies, relative to the predictions with no treatment of degeneracy.

Finally, as discussed above, a small fraction of the RIFs were predicted to originate in the hotspot. The hotspot plasma is a weakly coupled plasma for which the form of the stopping power is known. The hotspot has an additional contribution to RIF production from alphaparticle induced knock-on ions. Our simulations predict that the combination of the neutron-induced and alphainduced RIFS from the hotspot make up about 10-15\% of the measured ${ }^{167} \mathrm{Tm} /{ }^{168} \mathrm{Tm}$ signal. These RIF contributions are included in Table 1 .

\section{SUMMARY}

The measurements presented here represent the first observation of RIF neutrons in any inertial confinement 
fusion system. RIFs were observed in both Low Foot and High Foot design cryogenic capsules, with a magnitude of about $10^{-4}$ of the total neutron spectrum. The majority of the RIF neutrons were produced in the dense cold fuel surrounding the burning hotspot of the capsule, which is moderately to strongly coupled $(\Gamma \sim 0.3-1.2)$ and electron degenerate $\left(\theta_{\text {Fermi }} / \theta_{e} \sim 2.3-5.0\right)$. Thus, the RIF data presented here provide singular tests of stopping power under warm dense plasma conditions. Future direct measurements of RIF spectra produced in cryogenic NIF capsules by time-of-flight would place even more stringent constraints on energy loss in strongly coupled systems, particularly with respect to the effects of plasma degeneracy.
[1] M.J. Edwards et al., Phys. Plasmas 20, 070501 (2013).

[2] R.K. Fisher,P.B. Park, J.M. McChesney, and M.N. Rosenbluth, Nuclear Fusion 34, 1291 (1994), and J. Källne, L. Ballabio, J. Frenje, S. Conroy, G. Ericsson, M. Tardocchi, E. Traneus, and G. Gorini, Phys. Rev. Letts. 85, 1246(2000), and

L. Ballabio, G. Gorini, and J. Källne, Phys. Rev. E 55 , 3358 (1997), and

A. A. Korotkov, A. Gondhalekar, and R. J. Akers, Phys. of Plasmas 7, 957 (2000).

[3] The Relationship between Charged-Particle Fluence and Stopping Power, Gerard Jungman and A.C.Hayes, Los Alamos Internal Report, LA-UR-13-26171, (2013).

[4] G.Maynard and C. Deutsch, J. Physique, 46, 1113 (1985).

[5] G.B. Zimmerman, Recent Developments in Monte Carlo Techniques, Lawrence Livermore National Laboratory internal report, UCRL-JC-105616, (1990).

[6] Paul E. Grabowski, Michael P. Surh, David F. Richards, Frank R. Graziani, and Michael S. Murillo, Phys. Rev. Lett. 111, 215002, (2013).

[7] A.C. Hayes, Gerard Jungman, J.C. Solem, P.A. Bradley, and R.S. Rundberg, Modern Physics Letters A 21, No. 13 (2006) 1029.

[8] A.C. Hayes and Gerard Jungman, The Relationship between Knock-on Charged-Particle Reactions and Reaction-in-Flight Neutrons, Los Alamos Internal Report, LA-UR-13-27001, (2013).

[9] For the sake of brevity, we use the symbol $n_{D T}$ to mean both the deuteron and triton density. IF the knock-on ion is a deuteron, $n_{D T}$ represents the deuteron density in the expression for $Q_{0}$, and the density of the triton in eq. 1, and vise-verse.

[10] CPT-Implode is a 1-D ICF burn code based on the burn physics of 23. CPT refers to charged-particle transport as the code places and emphasis on knock-on ions and the production of RIFs.
[11] M. M. Marinak, G. D. Kerbel, N. A. Gentile, O. Jones, D. Munro, S.Pollaine, T. R. Dittrich, and S. W. Haan, Phys. Plasmas 8, 2275 (2001)

[12] L. S. Brown, D. L. Preston, and R. L. Singleton, Jr., Phys. Rep. 410, 237 (2005).

[13] Günter Zwicknagel, Theory and simulation of heavy ion stoping in plasma, Laser and Particle Beams 27 (2009) 399-413.

[14] A.C. Hayes, Accuracy of the BPS and Maynard-Deutsch Stopping Powers, Internal Los Alamos Report, LA-UR13-22639, (2013).

[15] T.R. Dittrich, O.A. Hurricane, D.A. Callahan, E.L. Dewald, T. Dppner, D.E. Hinkel, L.F. Berzak Hopkins, S. Le Pape, T. Ma, J.L. Milovich, J.C. Moreno, P.K. Patel, H.-S. Park, B.A. Remington, J.D. Salmonson, and J.L. Kline, Phys. Rev. 112, 055002 (2014), and

H.-S. Park, O.A. Hurricane, D.A. Callahan, D.T. Casey, E.L. Dewald, T.R. Dittrich, T. Dppner, D.E. Hinkel, L.F. Berzak Hopkins, S. Le Pape, T. Ma, P.K. Patel, B.A. Remington, H.F. Robey, J.D. Salmonson, and J.L. Kline, Phys. Rev. Lett. 112, 055001 (2014), and

O. A. Hurricane, et al., Phys. Plasmas, 21, 056314 (2014).

[16] C. K. Li and R. D. Petrasso, Phys. Rev. Lett. 70, 3059 (1993)

[17] A. C. Hayes, P. A. Bradley, G. P. Grim, Gerard Jungman, and J. B. Wilhelmy,PHYSICS OF PLASMAS 17, 012705,2010 .

[18] D.A. Shaughnessy, et al., Rev. Sci. Instrum. 83 , 10 D917 (2012).

[19] G.P. Grim, et al., SPIE conference proceedings, in press.

[20] Charles Cerjan, Paul T. Springer, and Scott M. Sepke, Phys. Plasmas 20, 056319 (2013).

[21] E.G. Corman, W.E. Loewe, G.E. Cooper, and A.M. Winslow, Nucl. Fus, 15377 (1975).

[22] L. Spitzer, The Physics of Fully Ionized Gases, New York: Interscience, (1965).

[23] J. Sanz, J. Garnier, C. Cherfils, B. Canaud, and L. Masse, M. Temporal, Phy. Plasmas 12, 11272005. 